\title{
Concept-Based Question Analysis for an Efficient Document Ranking
}

\author{
Seung-Eun Shin ${ }^{1}$, Young-Min $\mathrm{Ahn}^{2}$, and Young-Hoon Seo ${ }^{2}$ \\ ${ }^{1}$ Chungbuk National University BK21 Chungbuk Information Technology Center \\ Cheongju, Chungbuk, 361-763, Korea \\ seshin@nlp. chungbuk.ac.kr \\ ${ }^{2}$ School of Electrical \& Computer Engineering, Chungbuk National University \\ Cheongju, Chungbuk, 361-763, Korea \\ mania@nlp.chungbuk.ac.kr, yhseo@chungbuk.ac.kr
}

\begin{abstract}
This paper describes a concept-based question analysis for an efficient document ranking. Our idea is that we can rank efficiently documents containing answers for questions when we use well-defined concepts because concepts occurred in questions of same answer type are similar. That is, we can retrieve more relevant documents if we know the syntactic and semantic role of each word or phrase in question. For each answer type, we define a concept rule which contains core concepts occurred in questions of that answer type. Concept-based question analysis is a process which tags concepts to morphological analysis result of a user's question, determines the answer type, and extracts untagged concepts from it using a matched concept rule. Empirical results show that our concept-based question analysis can rank documents more efficiently than any other conventional approach. Also, concept-based approach has additional merits that it is language universal model, and can be combined with arbitrary conventional approaches.
\end{abstract}

Keywords: Concept, Concept rule, Question Analysis, Document Ranking, Question Answering.

\section{Introduction}

Information retrieval (IR) techniques used to find information fast and exactly from tremendous documents have been rapidly developed with the growth and commercial application of the Internet. However, we can often find that high ranked documents retrieved from a general web search engine may be far from a user intension. Therefore, effective retrieval and rank techniques are needed to provide more relevant documents to users or question answering (QA) systems are demanded for user's convenience.

The TREC 2005 QA track contained three tasks: the main question answering task, the document ranking task, and the relationship task. The goal of the document ranking task was to create pools of documents containing answers to questions. The task was to submit, for a subset of 50 of the questions in the main task, a ranked list of up to 1000 documents for each question [1]. 
Current research trends tend to focus on applying a natural language processing (NLP) technique for efficient document retrieval [2], [3], [4], [5]. However, such approaches cannot effectively reflect the meaning of sentences because they use only index terms extracted from the morphological analysis or n-gram method. Results of all IR systems include many non-relevant documents because the index cannot naturally reflect the contents of documents and because queries used in IR systems cannot represent enough information in user's question [6]. That is, the statistical IR model cannot understand a user's intention because it does not consider semantics of index terms. That is an essential reason of the inaccurate IR.

Question analysis in QA determines answer type corresponding to question type using named entities, answer type taxonomies, and ontologies such as WordNet in addition to question analysis in IR systems [7], [8], [9]. The number of answer types varies widely from single digits to a few thousands. The subdivided classification of answer type helps to extract more accurate answer by reducing the number of answer candidates in phase of answer extraction.

This paper describes a concept-based question analysis for an efficient document ranking in which concept rather than keyword makes an important role on document retrieval. We define concepts commonly occurred in the same type of questions, and use those concepts on document ranking to retrieve more relevant documents.

\section{Concept-Based Question Analysis}

Concept-based question analysis applies NLP techniques on a user's natural language questions and analyzes them semantically for an efficient document ranking. For each answer type, we define a concept rule which contains core concepts occurred in questions of that answer type. Concept is a well-defined semantic component for each answer type.

Let's consider following questions whose answer type is either an author or time.

(Question 1) Who wrote Hamlet?

(Question 2) Who is the author of the novel, "The old man and the sea"?

(Question 3) When was the American Legion founded?

(Question 4) When was Hong Kong returned to Chinese sovereignty?

We can see concepts to be used commonly to represent the information demand of users in questions (Question 1-2) whose answer type is an author. They are titles of books ("Hamlet", "The old man and the sea"), an interrogative pronoun (who), noun to express the author (author), verb to express the author (write), and noun to express the genre (novel). Concepts to be used commonly in questions (Question 3-4) whose answer type is time are objects of an event (American Legion, Hong Kong), verbs to represent an event (found, return), an interrogative pronoun (when), and nouns to be related with an event (Chinese, sovereignty).

The statistical model extracts index terms (Who, write, Hamlet) from (Question 1) and index terms (When, American Legion, found) from (Question 3). Then it generates queries using index terms and ranks relevant documents by the querydocument similarity. Therefore, it will retrieve the document which includes simply many index terms to more relevant document than the really relevant document which 
includes sentences such as "Shakespeare is the author of Hamlet". Besides, users consider the precision at top documents more important than the total precision because most IR systems offer results over the hundreds of thousands of documents. To solve this problem, we determine answer types and subtypes of answers, extract concepts from user's questions, and use them for an efficient document ranking.

A concept is not simple meaning of a word but the semantic role of a word or phrase in sentences and is used to represent the user's intention. We defined subtypes of the answer and concepts from 643 questions whose answer type is a person in TREC QA Data and Web, and we constructed a concept dictionary by tagging concepts manually on those questions and expanded it using a synonym dictionary.

Table 1 shows a sample of subtypes of the answer and concepts. Subtypes of the answer type 'person' are classified by 24 categories such as author, family, prizewinner, politician, developer, inventor, scholar, entertainer, player etc. We are currently defining 125 concepts.

Table 1. Sample of subtypes of the answer and concepts

\begin{tabular}{c|l}
\hline Subtype of the answer & \multicolumn{1}{c}{ Concepts } \\
\hline Common & Nationality, Time, Sex, Person, $\ldots$ \\
Author & Book_Title, Author Noun, Author Verb, ... \\
Family & Relationship, Base Person, Relationship Info, ... \\
Prizewinner & Prize, Prize Noun, Prize Verb, Ceremony/Place, ... \\
Politician & Position, Event, Organization, Election Noun, ... \\
\hline
\end{tabular}

Table 2. Sample of concept tags and concept dictionary

\begin{tabular}{|c|c|c|}
\hline Concept & Tag & Concept Dictionary \\
\hline $\begin{array}{c}\text { Clue Adverb } \\
\text { Time }\end{array}$ & $\begin{array}{l}\text { \%Adverb } \\
\text { \%Time }\end{array}$ & choi-cho(first), ma-ji-mak(last), ... \\
\hline Who & @Who & nu-gu(who) \\
\hline Person & @Person & sa-ram, in-mul, bun(person), \\
\hline Author Noun & @ Author_N & jeo-ja, jak-ga, geul-sseun-i(author), ... \\
\hline $\begin{array}{l}\text { Author Verb } \\
\text { Genre }\end{array}$ & @ Author_V & jeo-sul-ha, jeo-jak-ha, sseu(write), ... \\
\hline Relationship & @Relationship & a-deul(son), bu-in(wife), a-beo-ji, (father), ... \\
\hline Base Person & \#Base_Person & \\
\hline Book_Title & \#Book_Title & \\
\hline
\end{tabular}

Table 2 is a sample of concept tags and concept dictionary. Tag for each concept consists of 'Property' + 'Concept'. Properties are divided into three properties such as '\%', '@', '\#'. We are currently constructing the concept dictionary which includes 2,039 vocabularies.

Concepts with ' $\%$ ' property are placed relatively free in sentence, and so they are inappropriate to handle by rules. Some ' $\%$ ' property concepts such as ' $\%$ Adverb' may be extracted from concept dictionary, and others such as '\% Time' from rules. These concepts are used importantly in document ranking though they are not used in concept rule matching. 
Concepts with '@' property are extracted from concept dictionary. That is, we tag a word or phrase to '@' property concept if it is found in concept dictionary. Concepts with '\#' property are ones to be extracted only from concept rules. Proper nouns such as title of the book and person name belong to '\#' property concepts. '\#' property concepts are extracted from question after a concept rule is selected using '@' property concepts.

We defined a concept rule manually for each answer type to extract concepts from a user's questions. Such a concept rule is represented as concepts and grammatical morphemes in order to consider semantic and syntactic structure of the user's questions. Fig. 1 shows the BNF notation of the concept rule and characteristics of Korean considered in our discussion. The concept rule consists of a list of <word information> which consists of concepts and grammatical morphemes according to the answer type.

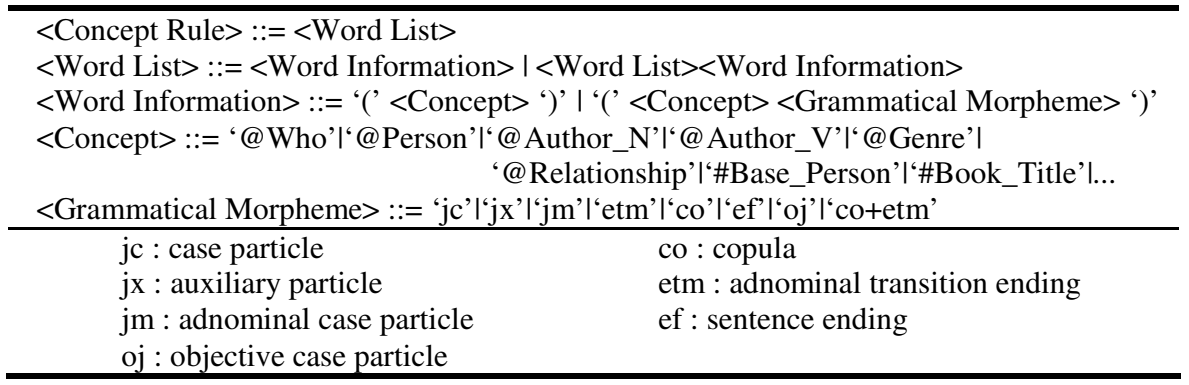

Fig. 1. BNF notation for the concept rule

The following examples are a concept rule for 'author' that is represented as an extended BNF and process of concept-based question analysis for (Question 2).

- Example of the concept rule for the author

3. (\#Book_Titleco+etm) (@Genrejm)(@Author_N jx?) (@Who)?

4. (\#Book_Title jc) (@Author_V etm) (@Personl@Author_Njx?) (@Who)?

5. (\#Book_Titlejm) (@Author_Njx?) (@Who)?

$<$ Concept-based question analysis for (Question 2) >

- "No-in-gua ba-da"ran so-seol-ui jeo-ja-neun nu-gu-ib-ni-gga?

(Who is the author of the novel, "The old man and the sea"?)

- Morphological analysis result of (Question 2) :

"No-in-gua ba-da"/nc+i/co+ra-go/ec+ha/pv+neun/etm so-seol/nc+ui/jm jeo$\mathrm{ja} / \mathrm{nc}+$ neun/jx nu-gu/np+i/co+ b-ni-gga/ef

- Word List:

(“No-in-gua ba-da"co+etm) (@Genrejm) (@Author_N jx) (@Who co+ef)

- Concept rule :

(\#Book_Title co+etm) (@Genre jm) (@Author_N jx?) (@Who)?

- Result of Concept-based Question Analysis for (Question 2) 
- Answer type : in-mul (Person)

- Subtype of the answer : jeo-ja (Author)

- Book_Title : No-in-gua ba-da (The old man and the sea)

- Genre : so-seol (novel)

We tag concepts to morphological analysis result of a user's question and construct the concept list. The concept list is matched by each concept rule. If there is a matched concept rule, we determine answer type and extract concepts of '\#' property using that concept rule. We select the longest rule when we have several matched concept rules.

If there is no concept rule to be matched to a user's questions, we extract concepts from the user's questions by following manner. We classified questions whose answer type is a person whether it contains a verb or not. The question which includes a verb consists of Event_V (verb to represent an event), Person (noun to represent a person), Property_N (noun to represent the property of a person such as doctor, author, and so on), and Who (interrogative). The question which does not include a verb consists of Property_N, NP (noun phrase), and Who. Therefore, we designed the common concept rule according to the syntactic structure of Korean natural language questions as follow.

- Common concept rule for the question which includes a verb

1. (\#NP jc) (\#Event_V etm) (@Personl@Property_N) (@Who)?

2. (\#NP jx) (@Who) (\#Event_V ef)

3. (@Who) (\#NP jc) (\#Event_V ef)

- Common concept rule for the question which does not include a verb

1. (\#NP jm) (@Property_N) (@Who)?

2. (\#NP jc) (@Property_N) (@Who)?

3. (@Property_N) (@Who)?

Although we cannot determine the subtype of the answer, we can extract concepts by using common concept rules from a user's question which has not the concept rule to be applied.

\section{Document Ranking}

We generate queries that reflect various syntactic structures to represent the answer and utilize them for an efficient document ranking. We can retrieve a document that includes generated queries as a relevant document and improve the precision of document retrieval. Other approaches that do not analyze the user's question semantically can hardly obtain queries whose syntactic structure is different from the user's question, but our approach can generate queries using the answer type and concepts as results of concept-based question analysis.

We designed query generation concept rules in order to generate queries, and it is made of concepts and grammatical morphemes. The query generation concept rules have concepts and syntactic structures used to represent an answer. We can generate 
queries using the query generation concept rules, concepts, concept dictionary, and synonym dictionary. Query generation concept rules for an author are examples of query generation concept rules.

- Query generation concept rules for an author

3. (Book_Title jm) (Author_N)

4. (Book_Title co+etm) (Genre oj) (Author_V)

We can generate queries using query generation concept rules and results of the concept-based question analysis of (Question 2) as below.

$<$ Examples of the query generation $>$

- Query generation concept rule 3 : (Book_Title jm) (Author_N)

- Generated queries : "No-in-gua ba-da"-ui jeo-jaljak-gal.......geul-sseun-i

(writerlauthor of "The old man and the sea")

- Query generation concept rule 4 : (Book_Title co+etm) (Genre oj) (Author_V)

- Generated queries :

"No-in-gua ba-da"-ra-neun so-seol-eul jeo-sul-haljeo-jak-hal...... Isseu

(writelwrotel...... Ito compose the novel, "The old man and the sea")

Our approach can generate queries which have the same meaning as the original question, but have different structures such as above example. Generated queries reflect the syntactic structures of almost all phrases which have answers for that question. We retrieve a document that includes generated queries as a relevant document because they consist of concepts and syntactic structures used to represent an answer.

Formula (1) is a transformation of the cosine coefficient to determine querydocument similarity when our approach is combined with the vector model [10]. We calculate the query-document similarity by using formula (1) to retrieve a document which includes generated queries as a relevant document.

$$
\begin{gathered}
\text { if } \vec{d}_{i} \cdot \vec{q}_{g e} \neq 0 \text { then } \operatorname{sim}\left(d_{i}, q_{u}\right)=\vec{d}_{i} \cdot \vec{q}_{g e} \\
\text { else } \operatorname{sim}\left(d_{i}, q_{u}\right)=\frac{\vec{d}_{i} \cdot \vec{q}_{u}}{\left|\vec{d}_{i} \| \vec{q}_{u}\right|} \\
d_{i}: \text { document, } q_{u}: \text { user's question, } \vec{d}_{i}: \text { document vector } \\
\vec{q}_{g e}: \text { generated query vector, } \vec{q}_{u}: \text { query vector by query expansion }
\end{gathered}
$$

We can rank documents by formula (1) because queries are generated by concepts and syntactic structures which are used to represent an answer. We can increase the precision at document retrieval by ranking documents which include a generated query in the high position. 


\section{Experimental Results}

We randomly selected 100 questions as a test set from natural language questions whose answer type is a person. They were questions which were used actually for IR in the Web. We measured the precision at $\mathrm{N}$ documents. In our experiments, we used Google and Yahoo as the IR systems and used only top 30 results of such systems for the precision at $\mathrm{N}$ documents.

Table 3 shows the accuracy of concept-based question analysis. Accuracy of concept extraction is (the number of concepts which are extracted correctly)/(the total number of concepts). Table 4 shows the precision at $\mathrm{N}$ documents of the document ranking result.

Table 3. Accuracy of concept-based question analysis

\begin{tabular}{c|c|c}
\hline & $\begin{array}{c}\text { Question which concept } \\
\text { frame is applied }\end{array}$ & $\begin{array}{c}\text { Question which common } \\
\text { concept rule is applied }\end{array}$ \\
\hline The number of questions & 69 & 31 \\
\hline $\begin{array}{c}\text { Accuracy of } \\
\text { answer type determination }\end{array}$ & 1.000 & 0.903 \\
\hline $\begin{array}{c}\text { Accuracy of } \\
\text { subtype determination }\end{array}$ & 1.000 & $\begin{array}{c}\text { We cannot determine the } \\
\text { subtype of the answer }\end{array}$ \\
\hline $\begin{array}{c}\text { Accuracy of } \\
\text { concept extraction }\end{array}$ & 0.918 & 0.845 \\
\hline
\end{tabular}

Table 4. Precision at $\mathrm{N}$ documents of the document ranking result

\begin{tabular}{c|c|c|c|c}
\hline \multicolumn{5}{c}{ Micro Averaging Precision } \\
\hline $\mathrm{N}$ & Google & $\begin{array}{c}\text { Google+ } \\
\text { Our approach }\end{array}$ & Yahoo & $\begin{array}{c}\text { Yahoo+ } \\
\text { Our approach }\end{array}$ \\
\hline At 3 docs & 0.584 & $0.803(+0.219)$ & 0.580 & $0.804(+0.224)$ \\
At 5 docs & 0.585 & $0.770(+0.185)$ & 0.557 & $0.743(+0.186)$ \\
At 10 docs & 0.548 & $0.646(+0.098)$ & 0.511 & $0.604(+0.093)$ \\
At 15 docs & 0.523 & $0.591(+0.068)$ & 0.478 & $0.541(+0.063)$ \\
At 20 docs & 0.489 & $0.541(+0.052)$ & 0.458 & $0.506(+0.048)$ \\
\hline
\end{tabular}

Precision at $\mathrm{N}$ documents: The percentage of documents retrieved in the top $\mathrm{N}$ that is relevant. If the number of documents retrieved is fewer than $\mathrm{N}$, then all missing documents are assumed to be non-relevant.

In case that our approach is applied to Google and Yahoo, the test of precision at $\mathrm{N}$ documents was improved by $+0.2215(\mathrm{~N}=3),+0.1850(\mathrm{~N}=5)$ and $+0.0955(\mathrm{~N}=10)$. If our approach is applied to more documents, the precision at $\mathrm{N}$ documents can be improved more than that of table 4 . In addition, we found that it is possible to make document ranking more efficient by analyzing questions based on concepts which are comparatively short but fully expressing a user's intentions. 


\section{Conclusion and Future Work}

In this paper, we proposed a concept-based approach for an efficient document ranking. Concept-based question analysis extracts concept components from morphological analysis result for a user's question, determines answer type, and generates queries using extracted concepts. And then, we rank document which include a generated query in the high position as a relevant document.

We applied our concept-based question analysis to document retrieval system, Google and Yahoo, and obtained a notable improvement $(+0.2215, \mathrm{~N}=3)$ in the precision at $\mathrm{N}$ documents. Although we make an experiment in the restricted domain in which questions require a person name as its answer, our concept-based approach can retrieve more relevant documents than any other conventional approach. Also, our approach has additional merits that it is a language universal model, and can be combined with arbitrary conventional approaches in the method that concept-based approach is used when the given question can be analyzed to one of the defined concept rules, and other approach is used otherwise.

We plan to expand concept rules for various domains, and expect incremental performance improvement.

Acknowledgments. This research was supported by the Ministry of Information and Communication, Korea under the Information Technology Research Center support program supervised by the Institute of Information Technology Assessment, IITA2006-(C1090-0603-0046).

\section{References}

1. Ellen M. Voorhees and Hoa Trang Dang: Overview of the TREC 2005 Question Answering Track. TREC 2005, (2005)

2. A.T. Arampatzis, T. Tsoris, C.H.A. Koster and Th.P. van der Weide: Phrase-based Information Retrieval. Journal of Information Processing \& Management, 34(6), (1998) 693-707

3. Boris V. Dobrow, N.V. Loukachevitch and T.N. Yudina: Conceptual Indexing Using thematic Representation of Texts. TREC-6, (1997)

4. Jose Perez-Carballo and Tomek Strzalkowski: Natural language information retrieval: progress report. Journal of Information Processing \& Management, 36(1), (2000) 155-178

5. C. Zhai: Fast Statistical Parsing of Noun Phrases for Document Indexing. In Proceedings of the Fifth Conference of Applied Natural Language Processing, (1997)

6. S. H. Myaeng: Current Status and New Directions of Information Retrieval Technique. Communications of the Korea Information Science Society, 24(4), (2004) 6-14

7. A. Ittycheriah, M. Franz, W. Zhu, A. Ratnaparkhi: IBM's Statistical Question Answering System. In $9^{\text {th }}$ Text Retrieval Conference, (2000) 229-334

8. S. Haragagiu, M. Pasca, S. Maiorano: Experiments with open-domain with open-domain textual question answering. In COLLING-2000, (2000) 292-298

9. M. Pasca, S. Harabagui: High Performance Question / Answer. In $24^{\text {th }}$ Annual International ACM SIGIR Conference on Research and Development in Information Retrieval, (2001) 366-374

10. G. Salton: Automatic Text Processing, Addison-Wesley, (1989) 